\title{
INTEGRATION OF LOCAL-GLOBAL UPSCALING AND GRID ADAPTIVITY FOR SIMULATION OF SUBSURFACE FLOW IN HETEROGENEOUS FORMATIONS
}

\author{
M. GERRITSEN* AND J. V. LAMBERS ${ }^{\dagger}$
}

\begin{abstract}
We propose a methodology, called multi-level local-global (MLLG) upscaling, for generating accurate upscaled models of permeabilities or transmissibilities for flow simulation on adapted grids in heterogeneous subsurface formations. The method generates an initial adapted grid based on the given fine-scale reservoir heterogeneity and potential flow paths. It then applies local-global (LG) upscaling for permeability or transmissibility [7], along with adaptivity, in an iterative manner. In each iteration of MLLG, the grid can be adapted where needed to reduce flow solver and upscaling errors. The adaptivity is controlled with a flow-based indicator. The iterative process is continued until consistency between the global solve on the adapted grid and the local solves is obtained. While each application of local-global upscaling is also an iterative process, this inner iteration generally takes only one or two iterations to converge. Furthermore, the number of outer iterations is bounded above, and hence the computational costs of this approach are low. We design a new flow-based weighting of transmissibility values in local-global upscaling that significantly improves the accuracy of LG and MLLG over traditional local transmissibility calculations.

For highly heterogeneous (e.g., channelized) systems the integration of grid adaptivity and localglobal upscaling is shown to consistently provide more accurate coarse-scale models for global flow, relative to reference fine-scale results, than do existing upscaling techniques applied to uniform grids of similar densities. Another attractive property of the integration of upscaling and adaptivity is that process dependency is strongly reduced, that is, the approach computes accurate global flow results also for flows driven by boundary conditions different from the generic boundary conditions used to compute the upscaled parameters.

The method is demonstrated on Cartesian Cell-based Anisotropic Refinement (CCAR) grids, but can be applied to other adaptation strategies for structured grids, and extended to unstructured grids.
\end{abstract}

1. Introduction. The permeability of reservoir rocks is often highly variable and uncertain. However, it can greatly affect flow and transport in subsurface formations, and must therefore be adequately modeled. The effects of uncertain reservoir heterogeneity can be included either through stochastic modeling or by simulating a number of deterministic geostatistical realizations of the reservoir [9], which is the underlying assumption in this paper. Because the computational costs of solving for a large number of realizations are high, simulations are generally performed on grids that are coarse compared to the given geological models. The grid size is typically determined by computational considerations. These coarsened flow models should adequately represent key behaviors, such as the overall flow rate for given boundary conditions or critical connected flow paths.

In this paper, we focus on single-phase upscaling to test the effectiveness of our new approach. Assuming single-phase, steady and incompressible flow in a heterogeneous reservoir, the governing dimensionless equation, assumed valid on the fine geological scale, is obtained by combining the continuity equation $\nabla \cdot \mathbf{u}=\mathbf{0}$ and Darcy's law given by $\mathbf{u}=-\mathbf{K} \cdot \nabla p$, to get

$$
\nabla \cdot(\mathbf{k} \cdot \nabla p)=0
$$

Here $p$ is the pressure, $\mathbf{u}$ the Darcy velocity and $\mathbf{K}$ the permeability tensor, all of which are assumed non-dimensionalized by appropriate reference values. Although

\footnotetext{
*Department of Energy Resources Engineering, Stanford University, Green Earth Sciences Building, Stanford, CA 94305-2220, USA

${ }^{\dagger}$ Department of Energy Resources Engineering, Stanford University, Green Earth Sciences Building, Stanford, CA 94305-2220, USA
} 
homogenization of equations with variable coefficients generally yields coarse-scale equations of a different form than the original fine-scale equations, it is common practice to keep the same equation as (1.1) for the pressure on the coarse scale, with the permeability tensor replaced by the coarse-scale permeability tensor $\mathbf{K}^{*}$, also referred to as the effective permeability tensor, and $p$ by the coarse-scale pressure. This has been found to lead to adequate coarse-scale pressure solutions $[10,27,5]$. We use Eqn. (1.1) indiscriminately of scale, and seek to find high quality representations of the global fine grid flow in the reservoir when the equation is solved on a coarse grid. The literature suggests three main approaches to control the quality of these representations: specialized upscaling, gridding and discretization strategies.

A large variety of single-phase upscaling techniques exist that are used to find representative permeability or transmissibility fields on the coarse grids. In purely local methods, the upscaled parameters are computed over fine-scale regions that correspond to the target coarse block under study. In extended local upscaling methods, regions neighboring the coarse block are included. In both approaches, the local flow is driven by generic Dirichlet, Neumann or periodic boundary conditions imposed on the fine-scale regions, see $[10,31,27]$. Although local methods may give satisfactory results for some reservoirs, they generally do not perform well when the spatial scales of important reservoir heterogeneities, such as high-permeability flow paths, are larger than the simulation grid, unless these grids are aligned with such paths. Global methods, which use global fine-scale flow simulations to determine coarse-scale parameters, can reduce errors in the presence of large connected flow paths, but they are generally expensive [19]. An attractive and relatively novel method to improve large-scale connectivity in upscaled permeabilities or transmissibilities is the local-global (LG) upscaling method [7]. Here, a standard extended local method is used to obtain an approximate coarse permeability or transmissibility field. Then, a coarse global solve is performed with generic boundary conditions. Interpolation of the coarse solution provides the Dirichlet boundary conditions for the next iteration of local solves. The process is repeated until the coarse field converges, which generally takes only a couple of iterations.

Gridding strategies include alignment of the grid with flow features and/or geological features, and local grid adaptation. Flow-aligned gridding, also referred to as flow-based gridding, in which grid cell boundaries are aligned with dominant flow paths, has been suggested by various authors, see, for example, [17] and references therein, to avoid smoothing of permeability contrasts straddled by the coarse grid. On such grids, local upscaling methods may lead to good representations of the reservoir heterogeneity. However, in strongly heterogeneous reservoirs, and especially in three dimensions, it is rather challenging to construct flow-based grids that are well aligned with the flow and have sufficient grid quality. An alternative is local grid adaptation strategies, in which the grid density is increased where needed to improve the representation of heterogeneity, for example, in regions with high permeability contrasts. Adaptive grids also allow important flow features with small spatial support, such as frontal regions, to be resolved accurately without sacrificing computational efficiency, see for example $[29,28,14]$. We think of adaptive grid strategies as part of the class of multi-scale methods, as discussed in, for example, [21] and references therein. Multiscale methods, designed to capture multiple scales involved in the reservoir fluid flow processes, include approaches that upscale to a coarse simulation grid and reconstruct the solution on a finer scale within each coarse grid cell using a functional representation, but also approaches based on grid refinement. Whether representing the flow on 
the fine-scale functionally or discretely, the success of the multi-scale method depends to a great extent on the chosen boundary conditions on the local fine regions.

Discretization strategies can also strongly impact the quality of the coarse solutions. With two-point flux approximations (TPFA), a flux through a given cell face is computed using pressure values from the cells that share the face, whereas with multipoint flux approximations (MPFA), additional cells may be involved. TPFA methods are attractive because of their simplicity, compactness and monotonicity. In many cases, the TPFA approximation is reasonable, but in cases with significant full-tensor effects, that may be introduced by the permeability field directly or by grid topology, larger errors should be expected, as assuming a TPFA is similar to neglecting the cross terms in upscaled permeability tensors. Interestingly, and perhaps surprisingly, TPFA methods combined with local-global procedures have been shown to provide good upscaled transmissibilities even in challenging cases $[7,6]$. It is generally agreed that when strong full-tensor effects are present, MPFA methods are preferable. Many authors have investigated MPFA approaches for solving single-phase flow problems with full-tensor permeability that is assumed constant over grid cells [1, 12, 22]. Naturally, MPFA methods lead to larger stencils than TPFA. On structured grids in three dimensions, for example, the MPFA O-method developed by Aavatsmark [1] results in a 27-point stencil for the cell-centered pressure equation, as compared to a 7-point stencil for TPFA. MPFA methods have been found to suffer from non-monotonicity; that is, the pressure solution may contain non-physical oscillations [26]. Various new MPFA methods have been suggested that improve their compactness and robustness, including the L-method by Aavatsmark et al. [2], and the spatially varying multipoint flux upscaling method, VCMP [23]. Preliminary studies combining VCMP with a local-global approach are also reported in this study.

1.1. This work: Integrating upscaling and adaptivity. The main questions we address in this work are

- how to combine local-global upscaling and local grid adaptivity to effectively represent large-scale heterogeneities on computational grids for single-phase flows,

- how to control the local grid adaptation during the upscaling process to improve the upscaling accuracy, and

- whether the proposed method reduces the process dependency that is generally introduced by upscaling methods.

While working on these research questions, we found that we can significantly improve local-global transmissibility upscaling by using a specialized flow-based weighting for local estimates of the transmissibilities for a given cell face. We will discuss this new weighting in this paper also.

We do not align the underlying computational grid with the flow, nor with the geology, instead focusing on the benefits that adaptivity and upscaling, specialized to adapted grids, can offer independently of any particular gridding strategy. For concreteness and simplicity, we apply the upscaling strategy to a cell-based anisotropic Cartesian refinement strategy. For similar reasons, we limit ourselves to TPFA methods. The proposed methodology is general enough, however, to be applicable to alternate gridding and discretization strategies. We focus on finite volume upscaling methods that lead to a cell-centered, coarse-scale pressure equation and conservative fluxes.

We continue the paper in Section 2 by describing Cartesian cell-based anisotropically defined (CCAR) grids and discretization of the single-phase pressure equation 
on such grids. In Section 3, we present the general Multi-Level Local-Global (MLLG) upscaling strategy that we propose in this paper for permeability and transmissibility upscaling, and discuss in more detail several components of this algorithm. We present an indicator that we use to adapt the grid during the MLLG iteration to reduce flow solver and upscaling errors. The new transmissibility weighting is also described in this section. Numerical results are presented in Section 4. We apply our algorithm to a suite of two-dimensional test problems, including four channelized domains, and with flow driven in several directions. Although the numerical results are given in two dimensions only for ease of visualization, the method is fully three dimensional, see also [15]. Discussion and conclusions are presented in the last two sections.

2. Solving the Single-Phase Pressure Equation Using An Adaptive Cartesian Approach. In this section, we describe the strategies for gridding and discretization that we will use in conjunction with our upscaling method.

2.1. Cartesian Cell-based Anisotropically Refined (CCAR) grids. When designing an efficient, adaptive simulation approach, a grid topology must ideally facilitate fast transitions from dense to coarse grids without sacrificing grid and solver quality, allow efficient computation despite potentially high levels of refinement, and lead to fast grid generation. We have found a Cartesian grid topology with anisotropic cell-based refinement and coarsening quite attractive because it allows aggressive grid adaptation while maintaining grid quality. We use adaptivity, rather than flow-based gridding, to align the grid with important flow and geological features and assess its effectiveness in ensuring flow accuracy. The CCAR grid is formed by a number of anisotropic refinements of its cells; i.e., splitting a cell in half along a coordinate direction, to construct a suitably refined mesh. This is illustrated for three refinement levels in Figure 2.1.
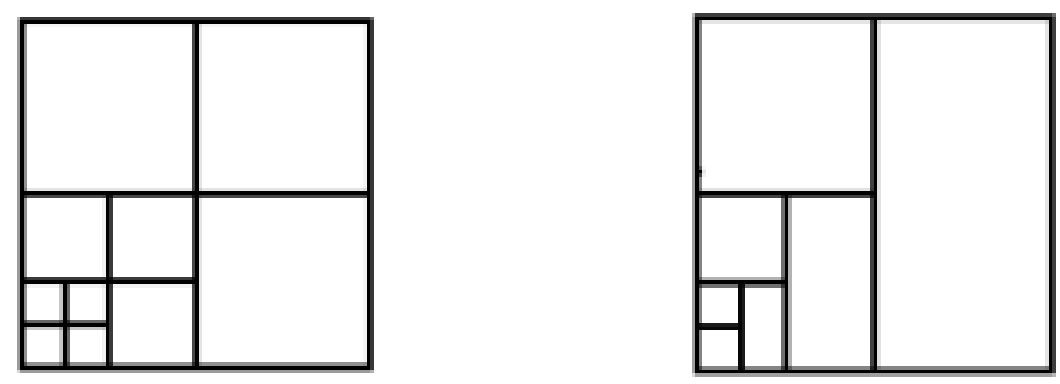

FIG. 2.1. CCAR grid cells with 3 levels of refinement. Left: isotropic refinement. Right: anisotropic refinement.

We impose the restriction that no cell can have more than four neighbors in each direction (or two neighbors in two dimensions) to maintain solution accuracy across interfaces [3]. The CCAR grids are stored using the unstructured data approach first suggested in [16], which leads to efficient look-ups. In this work, we denote cells in a CCAR grid, $G$, by $C_{i}, i=1, \ldots, N_{\text {cells }}$, with $N_{\text {cells }}$ the number of cells in $G$, and cell 
faces by $F_{i}, i=1, \ldots, N_{\text {faces }}$, with $N_{\text {faces }}$ the total number of faces in $G$.

We use the compact finite volume discretization of the pressure equation 1.1 on CCAR grids developed in [15]. For homogeneous media, this discretization is formally second order. For heterogeneous media, the computation of the flux through a face with a hanging node requires careful consideration.

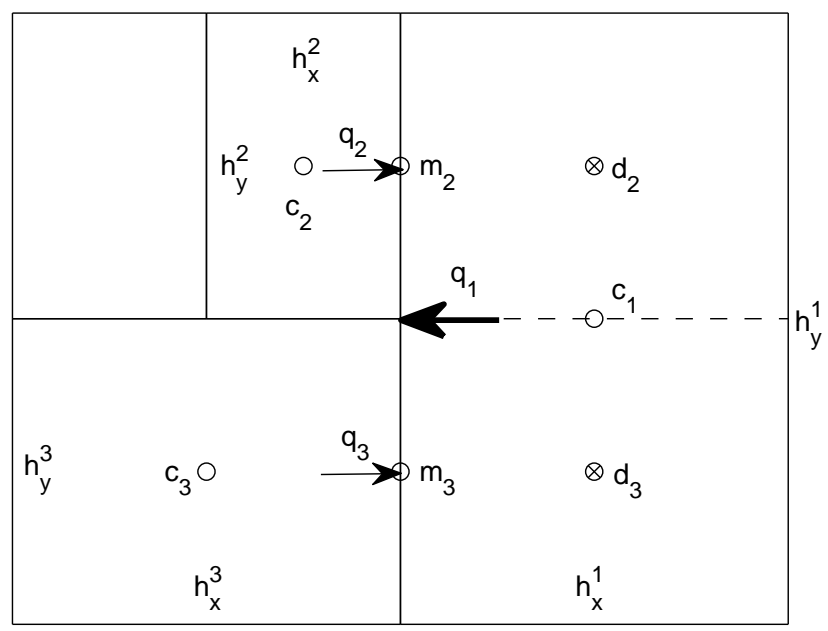

FIG. 2.2. The points $c_{j}, j=1,2,3, d_{j}, j=2,3$ and $m_{j}, j=2,3$, are used to compute the fluxes $q_{2}$ and $q_{3}$ across the half-faces between cell 1 and cell 2, and between cell 2 and cell 3.

Consider Figure 2.2, showing three cells $C_{i}, i=1,2,3$, with dimensions $h_{x}^{i}$ and $h_{y}^{i}$, and diagonal permeability tensors with diagonal elements $k_{x}^{i}$ and $k_{y}^{i}$. We will construct a flux for the face of cell 1 bordering cells 2 and 3 . The area of the face of cell $i$ that borders this face is denoted by $A_{i}$. The average pressures in the cells i are denoted by $p_{1}, p_{2}$ and $p_{3}$.

Requiring continuity of the flux across the half-faces, we obtain the equations

$$
\frac{k_{x}^{j}}{h_{x}^{j}}\left(p\left(m_{j}\right)-p\left(c_{j}\right)\right)=\frac{k_{x}^{1}}{h_{x}^{1}}\left(p\left(d_{j}\right)-p\left(m_{j}\right)\right), \quad j=2,3,
$$

where the points $m_{j}, c_{j}$ and $d_{j}$ are as given in the figure, and the corresponding fluxes

$$
q_{j}=\frac{2 h_{y}^{j}}{\frac{h_{x}^{1}}{k_{x}^{1}}+\frac{h_{x}^{j}}{k_{x}^{j}}}\left(p\left(d_{j}\right)-p\left(c_{j}\right)\right), \quad j=2,3 .
$$


We represent the pressure at the auxiliary points $d_{2}$ and $d_{3}$ in terms of the available pressures at nearby cell centers. To that end, we use a convex combination of two interpolations, one with a vertical orientation and one with a horizontal orientation, which are illustrated in Figure 2.3. For the vertically-oriented interpolation in $d_{2}$, we use bilinear interpolation at $c_{1}$, and the two closest cell centers besides $c_{2}$ and $c_{3}$, that form a triangle containing $d_{2}$. For the horizontally-oriented interpolation of $d_{2}$, we use bilinear interpolation at $c_{1}, c_{2}$, and the closest cell center that completes a triangle containing $d_{2}$. Similar triangles are constructed for $d_{3}$. We combine the horizontal and vertical interpolations with a weighting determined by the angle of the pressure gradient through the point $d_{i}, i=2$ or 3 , at which we are interpolating. This introduces a natural flow-based interpolation, and the resulting, wider, stencil at hanging nodes is reminiscent of the L-scheme suggested in [2].

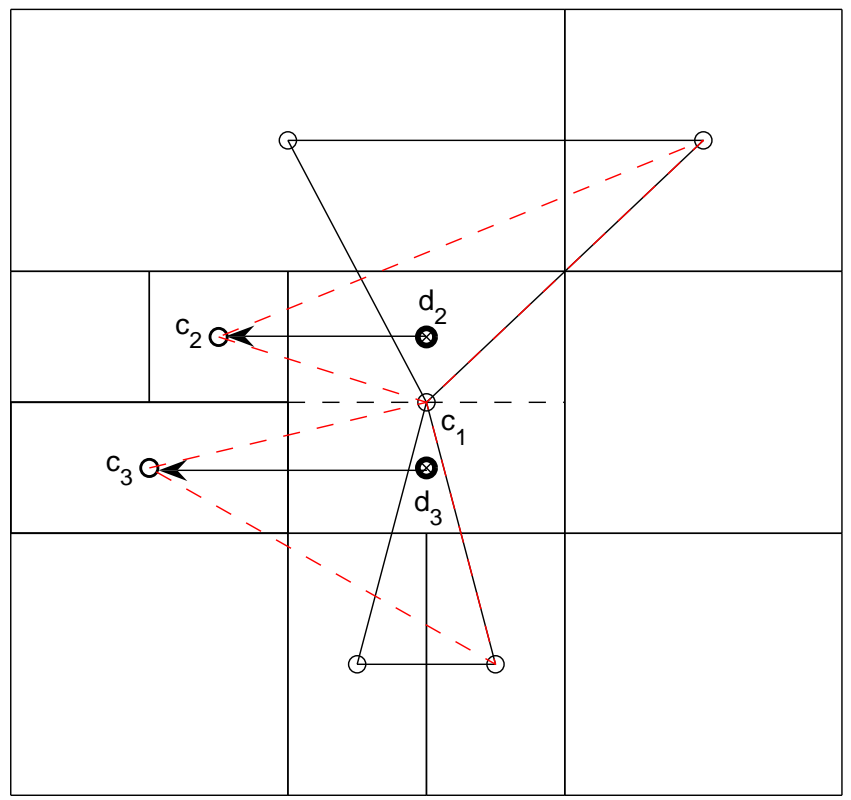

FIG. 2.3. Triangulations used to represent the pressure at $d_{2}$ and $d_{3}$ in terms of cell centers. The values at these points are obtained by bilinear interpolation of the values at the vertices of the horizontally, or vertically, oriented triangles containing them.

The resulting matrix remains sparse with a reasonable condition number thanks to the compact finite volume discretization. Because we do not have a strict TPFA discretization at hanging nodes, the matrix is not guaranteed to be an M-matrix and hence positivity is not ensured, but the number of matrix elements with the wrong signs is typically very small. Solution of the system using an algebraic multigrid solver 
(BoomerAMG [18]) is fast with almost linear convergence properties for our test cases.

3. Effective upscaling for CCAR grids. As mentioned in the introduction, it has been shown that for Cartesian grids, the local-global (LG) method leads to improved results as compared to local, or extended local, methods [7]. For each coarse grid cell, effective permeabilities or face transmissibilities are computed by solving a local flow problem on the fine geo-cellular grid directly around the coarse cell. This local flow problem is driven by boundary conditions interpolated from a coarse global solve. The resulting coupling between the local flow problems and the coarse global solve leads to an iterative scheme that generally converges rapidly. Because of its effectiveness, LG upscaling serves as the cornerstone of the local-global upscaling algorithm for CCAR grids, which we now present.

(a)
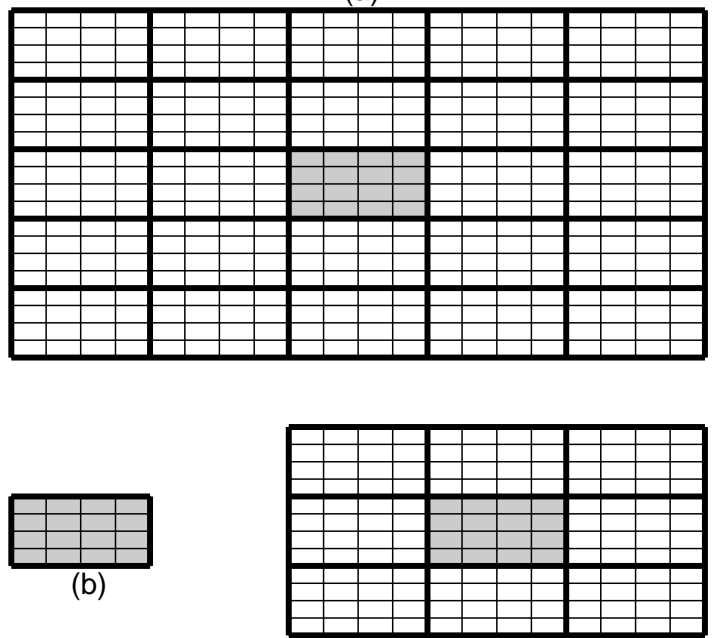

(c)

FIG. 3.1. Schematic showing (a) fine- and coarse-scale grids, (b) fine-scale local region and (c) fine-scale extended local region, with $r=1$ for permeability upscaling. For transmissibility upscaling, the fine-scale local region is centered around a cell-face rather than the cell itself.

3.1. The general Multi-Level Local-Global algorithm. In permeability upscaling, we associate with each cell $C_{i}, i=1$, in the CCAR grid $G$ an extended region $E_{i}=C_{i} \cup B_{i}$, where $B_{i}$ is a border region of constant width $r$ around $C_{i}$. In transmissibility upscaling the (extended) local region is centered around a coarse cell face $F_{i}$, rather than the cell itself. In each region $E_{i}$ we discretize the pressure equation on the uniform geocellular grid, which here we assume to be Cartesian. We note that in the local-global upscaling method in [7] the width of each dimension of the border region is chosen to be a multiple of the dimension of the corresponding grid cell instead. This model works well on a uniform grid, but in a CCAR grid, the coarsest cells do not such a large border because the fine-scale permeability within such cells tends to 
be smoother relative to the coarse grid scale. In permeability upscaling our border region scaling is also attractive for efficiency reasons: if a cell $C_{i}$ with corresponding extended region $E_{i}$ should be bisected during refinement, the resulting new cells will have extended regions whose union coincides with $E_{i}$. This allows pressure values on the boundary of $E_{i}$ to be re-used for the new regions.

The general MLLG algorithm is given as:

Construct the base CCAR grid $G$ using static refinement indicators

while grid adaptation not completed do

while not converged do

for each cell $C_{i}$ (permeability upscaling) or each face in $G$ do

Select boundary conditions on $E_{i}$ :

if this is the first iteration then

Use generic boundary conditions: constant pressure in one coordinate direction, no-flow in the other coordinate direction else

Use bilinear interpolation of global solutions $p_{x}$ and $p_{y}$ to approximate pressure on the boundary of $E_{i}$

Use Dirichlet boundary conditions using these pressure values end

Solve the pressure equation on $E_{i}$ with selected boundary conditions

Compute fluxes between the fine grid cells in $E_{i}$ and pressure values at their cell centers

Use these fluxes and pressure values on $E_{i}$ to compute new diagonal permeability

end tensor $K_{i}$ for $C_{i}$, or a new transmissibility for $F_{i}$

Solve generic flow problems on $G$ to obtain pressure fields $p_{x}$ and $p_{y}$

done

Check for convergence

Check if any of the cells in $G$ must be refined

done

The first step in the MLLG algorithm is the construction of an initial base CCAR grid. This base grid is designed with the aim of preserving important high permeability flow paths. After the base grid is generated, we apply local-global upscaling, as in the Cartesian case, to find representative permeability values on the cells $C_{i}$ of the CCAR grid, or transmissibility values for the faces $F_{i}$ of the CCAR grid. Once the local-global step has converged, which generally only takes one or two iterations, we check if further grid refinement is necessary, and repeat the local-global iteration on the updated grid. Thus, the MLLG algorithm has an inner iteration, which is a single application of local-global upscaling for CCAR grids, and an outer iteration with the number of outer iteration steps bounded above by the number of levels of refinement between the coarsest scale of the base grid, and the scale of the fine grid.

In the next subsection we discuss the main components of the MLLG algorithm in more detail.

\subsection{Main Components of the MLLG Algorithm.}

3.2.1. Creation of the Base CCAR Grid. To create a base CCAR grid, we apply a static refinement indicator based on the method proposed by Younis and Caers in [32]. The method is based on a "leaky" connected-set approach that 

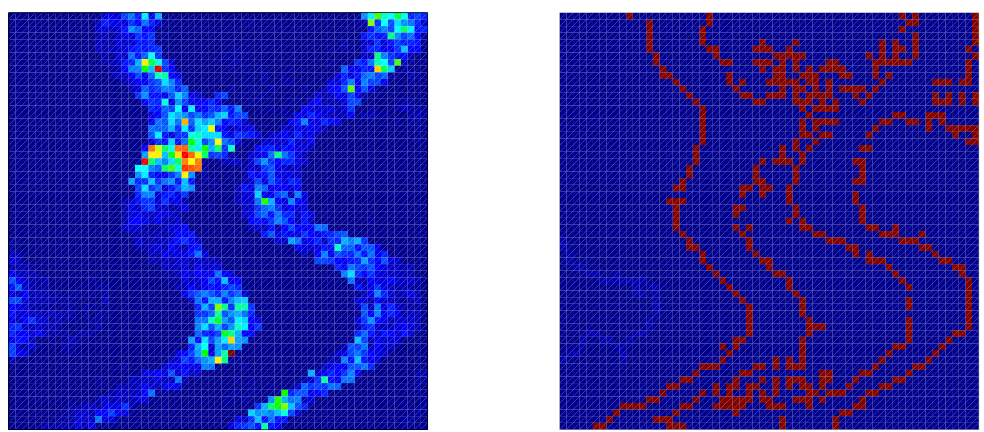

FIG. 3.2. Permeability field (left), and boundaries of connected flow paths detected by leaky connected-set approach (right)

delineates clusters of fine cells with similar permeability. Heuristic measures are used to color each connected set, indicating the level of the importance of capturing the set by refinement. Refinement flags are then obtained by explicit boundary detection. An illustration of the process appears in Figure 3.2. In the current implementation, a minimum level of refinement is set based on the resolution of the geocellular grid and efficiency considerations, and a CCAR grid $G$ is generated by iteration until these level constraints are met. Such a grid is illustrated in Figure 3.3.

In our experience, this approach has given us reasonable CCAR grids to start our MLLG process. We note that the grid is further adapted during MLLG.

3.2.2. Boundary Data for Local Solves. For each cell $C_{i}$ of the CCAR grid $G$, we must solve local flow problems (in two dimensions) over the corresponding extended region $E_{i}$. In order to obtain the Dirichlet boundary data for each such problem, we use bilinear interpolation, with the interpolation points obtained from each of the global coarse-scale solutions computed during the previous iteration of local-global upscaling. While it is possible to use a higher-order interpolation scheme, it is not advisable to do so because the interpolant would exhibit oscillatory behavior, as opposed to the monotonicity we would expect from a pressure field.

We illustrate the interpolation for permeability upscaling. In Figure 3.4, a cell $C_{i}$ is shown, along with its extended region $E_{i}$. For each of the points $p_{i j}, j=1,2, \ldots, 8$ along the boundary of $E_{i}$, we find the three cells of $G$ whose centers are as close to $p_{i j}$ as possible, and form a triangle containing $p_{i j}$. We then use barycentric coordinates [30] to approximate the pressure at $p_{i j}$.

3.2.3. Local Flow-based Refinement of the Base Grid. The fluxes across a face, from each global pressure solve, can be used to determine whether cells bordering the face should be refined.

Specifically, let $F_{i}$ be a face with area $A_{i}$, and let $q_{i}$ be the flux across the face obtained by a pressure solve on the CCAR grid with flow driven in the direction perpendicular to the face. If $Q$ is the global flow, and $A$ is the area of the face of the global flow domain perpendicular to the direction of the flow, then we determine that the cells sharing the face should be refined if

$$
\left|q_{i}\right|>\alpha \frac{A_{i}}{A}|Q|,
$$




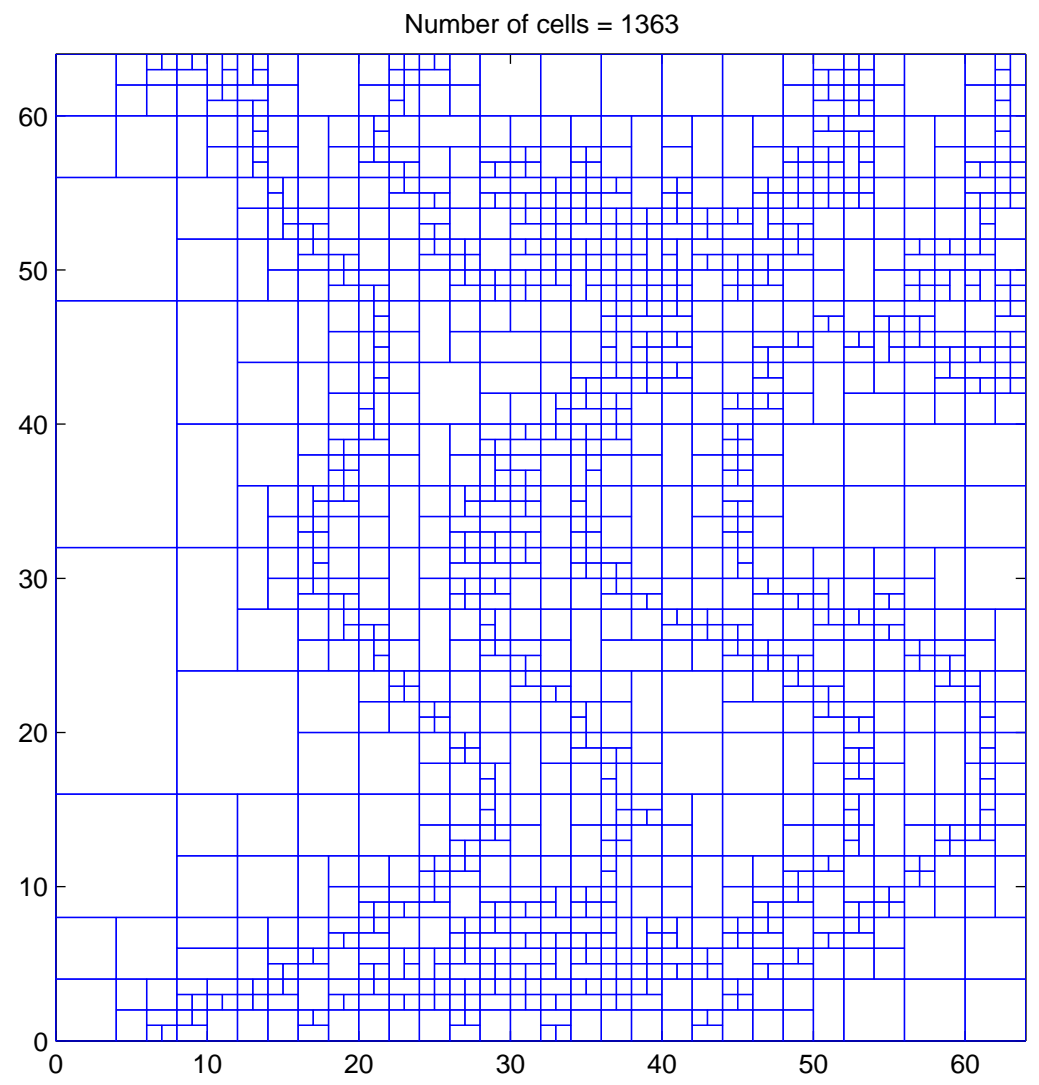

FIG. 3.3. base CCAR grid generated from permeability field and connected sets from Figure 3.2

where $\alpha$ is a proportionality constant that depends on the ratio of areas, i.e. $\alpha=$ $\alpha\left(\frac{A_{i}}{A}\right)$. The term $A_{i}|Q| / A$ can be interpreted as the face's "fair share" of the global flow.

To choose $\alpha\left(A_{i} / A\right)>1$, we use the following heuristics. First, when $A_{i}$ is the area of a face of a cell at the coarsest scale in the grid, then $\alpha\left(A_{i} / A\right)$ should be approximately 1 , so that refinement will always take place except in areas that are receiving less than their fair share of flow. This low tolerance is necessary to avoid overlooking high-flow paths that occur within large regions that otherwise have very low flow. On the other hand, as $A_{i}$ approaches the size of a face in the fine grid, then $\alpha\left(A_{i} / A\right)$ should be approximately $A / A_{i}$, so that refinement will not take place unless there is very high flow; that is, nearly all of the global flow is crossing the face $F_{i}$. This progression from low to high tolerance is used to avoid superfluous refinement while not missing any finer-scale regions of high flow. Under the assumption that $\alpha\left(A_{i} / A\right)=C\left(A_{i} / A\right)^{-p}$ for some positive real number $p$, we obtain

$$
\alpha\left(A_{i} / A\right)=\frac{\delta}{\left(A / A_{c}\right)^{p}}\left(\frac{A_{i}}{A}\right)^{-p},
$$




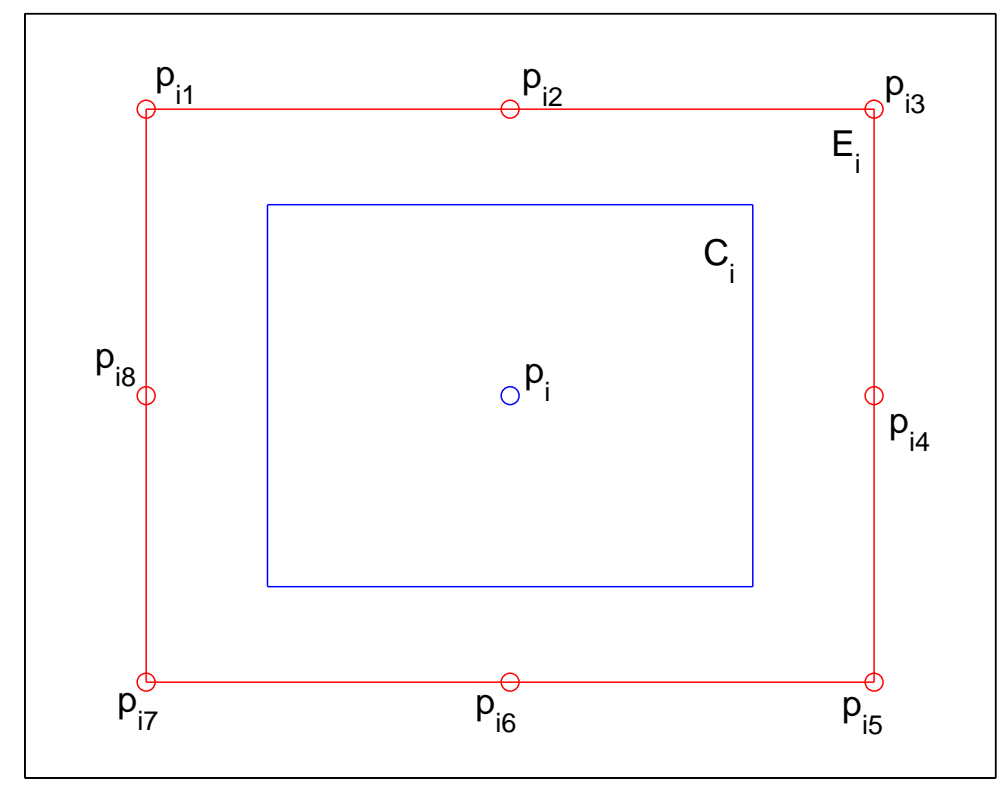

FIG. 3.4. points $p_{i j}, j=1,2, \ldots, 8$ on the boundary of an extended region $E_{i}$, shown for permeability upscaling, at which global coarse-scale pressure is interpolated. The region $E_{i}$ corresponds to the cell $C_{i}$ with center $p_{i}$.

where $A_{c}$ is the area of the largest face in the grid parallel to $F_{i}, \delta \approx 1$ is the chosen value of $\alpha\left(A_{c} / A\right)$, and $p$ is given by

$$
p=\frac{\ln \frac{A}{\delta A_{f}}}{\ln \frac{A_{c}}{A_{f}}},
$$

where $A_{f}$ is the area of each fine grid face that is parallel to $F_{i}$.

If the face $F_{i}$ borders only two cells, then both are refined. Otherwise, the face borders two hanging cells and one non-hanging cell; only the non-hanging cell is refined (e.g., cell 1 in Figure 2.2). All refinement is in the direction that is orthogonal to the face.

We can use additional refinement criteria to reduce the number of "hanging" nodes that occur in high-flow regions. First, we apply the above flow-based criterion to each "half-face" that is shared by a hanging cell and a non-hanging cell. If the flow across this half-face is more than its fair share, then the non-hanging cell is refined along the direction that is orthogonal to the face. This refinement also takes place if too much of the flow across the entire face $(75 \%$, in practice) is through one hanging node.

We note that in transmissibility upscaling, we also refine if the estimated transmissibility for a face is negative. This is further discussed in the next subsection.

3.2.4. A New Local Gradient-based Weighting of Transmissibilities. Referring back to Figure 2.2 we choose the extended region $E_{i}$ centered at the face 
$F_{i}$ for which we compute the flux to be large enough to contain the cells centered at the points $c_{1}, c_{2}$ and $c_{3}$. We solve the pressure equation on $E_{i}$ with Dirichlet boundary conditions as in permeability upscaling. The computation of the fluxes given by equation 2.2 from the local solution, along with the approximate pressure differences $\Delta p_{j}=p\left(d_{j}\right)-p\left(c_{j}\right)$ obtained by averaging the local pressure field over the appropriate regions, can be used to compute the transmissibilities $T_{2}$ and $T_{3}$ corresponding to the fluxes $q_{1}$ and $q_{2}$ indicated in Figure 2.2. These transmissibilities are then used in the stencils centered at $c_{2}$ and $c_{3}$. In the stencil centered at $c_{1}$, we use the transmissibility $T_{1}=T_{2}+T_{3}$.

The original local-global upscaling algorithm in [7] computed upscaled transmissibility across a face by considering only data obtained from a global flow driven by a pressure difference in the direction perpendicular to the face. But, since we are using two generic flow problems, we actually obtain two fluxes for each face. These fluxes should indeed not have equal weight: a flow that is nearly parallel to the face should not contribute much, if anything, to the computed face transmissibility. If it does, this may result in a stronger process dependency, which we would like to avoid. Rather than choosing only one flow, we designed a new weighting process for the transmissibilities, which we now describe.

Consider the simple case of a face shared by only two cells. Let $q^{(1)}$ and $q^{(2)}$ denote the fluxes across the face obtained from the two local pressure solves, obtained either in an extended local or a local-global method, and let $\Delta p^{(1)}$ and $\Delta p^{(2)}$ denote the differences in the cell averages of the pressure from these solves. From these values, we obtain two approximations for the transmissibility,

$$
T^{(j)}=-\frac{q^{(j)}}{\Delta p^{(j)}}, j=1,2 .
$$

Let $\mathbf{n}$ be a unit vector that is perpendicular to the face. We find a unique transmissibility $T$ using weighted least squares, with the weights determined by the average pressure gradients near the face, $\nabla p^{(1)}$ and $\nabla p^{(2)}$, where the averages may be cell or line averages (it remains to be determined which approach is best). This approach yields the system $W A T=W \mathbf{b}$, where

$$
W=\left[\begin{array}{cc}
\omega^{(1)} & 0 \\
0 & \omega^{(2)}
\end{array}\right], \quad A=\left[\begin{array}{c}
\Delta p^{(1)} \\
\Delta p^{(2)}
\end{array}\right], \quad \mathbf{b}=-\left[\begin{array}{c}
q^{(1)} \\
q^{(2)}
\end{array}\right]
$$

and

$$
\omega^{(i)}=\frac{\left|\nabla p^{(i)} \cdot \mathbf{n}\right|}{\left\|\nabla p^{(i)}\right\|}, \quad i=1,2 .
$$

The rationale for choosing the weights $\omega^{(i)}$ in this manner is to determine, in an average sense, the orientation of the flow from the two local solves. If either of these flows is determined to be nearly parallel to the face, then it is not be assigned much significance in the computation of the transmissibility across the face. On the other hand, if the flow is nearly perpendicular to the face, then the ratio of the negative flux to the pressure difference is believed to represent the coarse-scale transmissibility accurately.

The weighted least squares problem has the solution

$$
T=\frac{A^{T} W^{T} W \mathbf{b}}{A^{T} W^{T} W A}=-\frac{\left[\omega^{(1)}\right]^{2} q^{(1)} \Delta p^{(1)}+\left[\omega^{(2)}\right]^{2} q^{(2)} \Delta p^{(2)}}{\left[\omega^{(1)}\right]^{2}\left[\Delta p^{(1)}\right]^{2}+\left[\omega^{(2)}\right]^{2}\left[\Delta p^{(2)}\right]^{2}} .
$$


In the case of a face adjacent to hanging nodes, we apply this same approach to compute the transmissibilities across the half-faces. If either of the equations in (3.2) would yield a negative transmissibility, then it is not included in the computation of $T$. If both equations would yield negative transmissibilities, then we set $T$ equal to the sum of the fine-scale transmissibilities along the face, and flag the cells sharing the face for refinement.

We are able to prevent most instances of negative transmissibility from arising in the first place by computing flux across each face as follows. Assume that a face $F_{i}$ in the coarse grid consists of $n$ fine-scale faces $f_{j}, j=1, \ldots, n$. We compute the flux using

$$
q_{i}=\sum_{j=1}^{n} T_{j} \Delta p_{j},
$$

where, for each $j, T_{j}$ is the transmissibility across $f_{j}$, and $\Delta p_{j}$ is the pressure difference across the face. To compute this pressure difference, we first use the pressure values in the two fine-scale cells $c_{j}$ and $d_{j}$ bordering $f_{j}$. Then, we compute a pressure difference $\Delta p_{j}^{\prime}$ using the pressure values in the two cells $c_{j}^{\prime}$ and $d_{j}^{\prime}$ that are one cell removed from $f_{j}$ along the direction orthogonal to $f_{j}$. If $\Delta p_{j}$ and $\Delta p_{j}^{\prime}$ have opposite sign, then we assume that $\Delta p_{j}$ is an isolated fluctuation and compute the flux across $f_{j}$ using $\frac{1}{3} \Delta p_{j}^{\prime}$; otherwise we use $\Delta p_{j}$. The cells involved in the computation are shown in Figure 3.5.

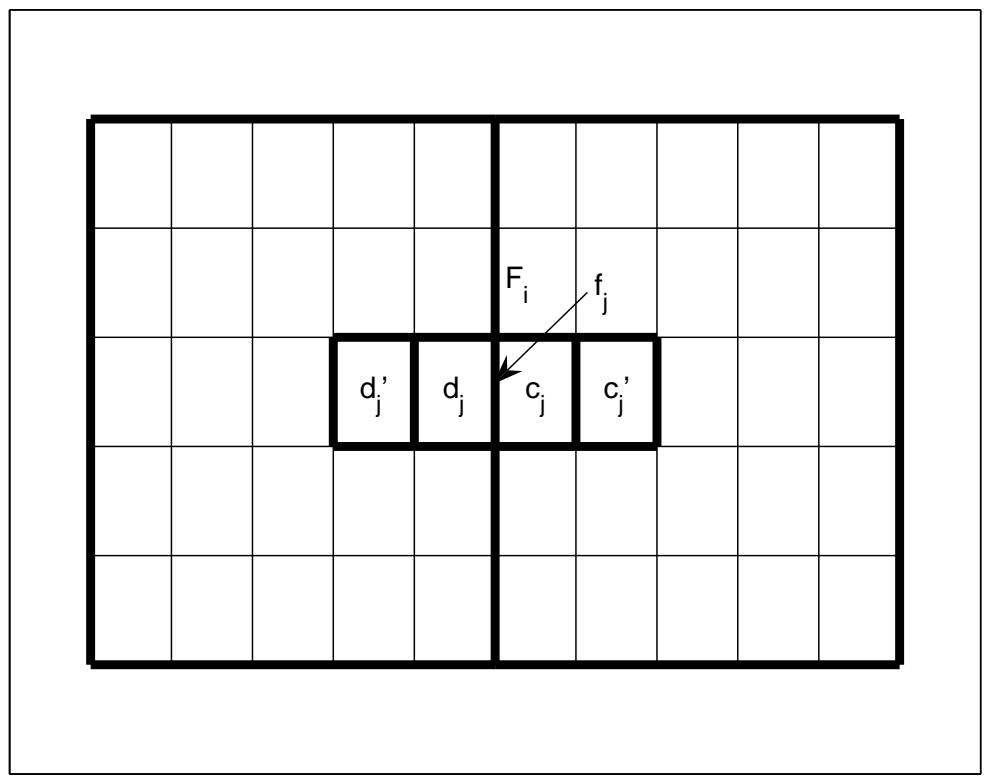

FIG. 3.5. The cells $c_{j}, d_{j}, c_{j}^{\prime}$ and $d_{j}^{\prime}$ used to compute the pressure differences $\Delta p_{j}$ and $\Delta p_{j}^{\prime}$ across the face $f_{j}$ that is a portion of the face $F_{i}$, the flux across which is used to compute the upscaled transmissibility across $F_{i}$. 
4. Numerical Results. In this section, we demonstrate the effectiveness of our upscaling method on a variety of permeability fields and boundary conditions.

\begin{tabular}{|l|l|}
\hline Abbreviation & Description \\
\hline EL & Extended local, on uniform Cartesian grid \\
\hline EL-W & EL with new transmissibility weighting \\
\hline LG & Local-global, on uniform Cartesian grid \\
\hline LG-W & Local-global with new transmissibility weighting, on uniform Cartesian grid \\
\hline EL-CCAR & Extended local, on CCAR grid \\
\hline MLLG & Multi-level local-global, on CCAR grid \\
\hline MLLG -W & Multi-level local-global with new transmissibility weighting, on CCAR grid \\
\hline
\end{tabular}

4.1. The Test Suite. Our test suite consists of three types of fine permeability fields that are used in the experiments described in this section. All have dimension $L_{x} \times L_{y}$, where $L_{x}=256$ and $L_{y}=64$, and uniform grid step size 1 in both spatial dimensions. The fields are:

- 10 realizations with long correlation lengths of $\ell_{x}=50, \ell_{y}=10$, aligned with the grid.

- 10 realizations with long correlation lengths of $\ell_{x}=75, \ell_{y}=5$, not aligned with the grid

- Four channelized domains, given by layers $44,48,76$ and 80 of the SPE10 Comparative Project (see [8]) that are modified to fit the domain dimensions $L_{x}=256$ and $L_{y}=64$.

Examples of realizations for the permeability fields with long correlation lengths are shown in Figure 4.1. For these cases, which are generated using 2-point statistics, we use mean 3.0 and standard deviation 1.735 , which corresponds to a log-normal distribution. The fine-scale permeability fields of the channelized domains are shown in Figure 4.2.

The main purpose of the realizations with long correlation lengths is to show the effectiveness of the new transmissibility upscaling obtained with the new weighting scheme. The results also show the accuracy of local-global upscaling vs. extended local upscaling, but we refer to [7] for more extensive comparisons. For these cases, we use the upscaled transmissibilities to solve the pressure equation on a Cartesian grid with 32 cells in the $x$-direction, and 16 cells in the $y$-direction. The dimensions of the cells are $\Delta x=8$ and $\Delta y=4$.

The channelized cases are used to assess the effectiveness of MLLG and MLLGW. Channelized reservoirs are generally difficult to upscale accurately because of the strong and large-scale flow paths. For the channelized cases, we use a CCAR grid obtained by iterative refinement of an initial $8 \times 4$ uniform Cartesian grid, with $\Delta x=32$ and $\Delta y=16$. The refinement is performed according to the criteria described in Section 3.2.3.

For each permeability field and each grid, we solve the pressure equation with the following six sets of boundary conditions, in order to measure how effective each algorithm is in reducing process dependency resolving the fine-scale velocity:

- Constant pressure/no-flow boundary conditions in the $x$ - and $y$-directions, respectively. For the first set, we prescribe $p(0, y)=1, p\left(L_{x}, y\right)=0$, and 

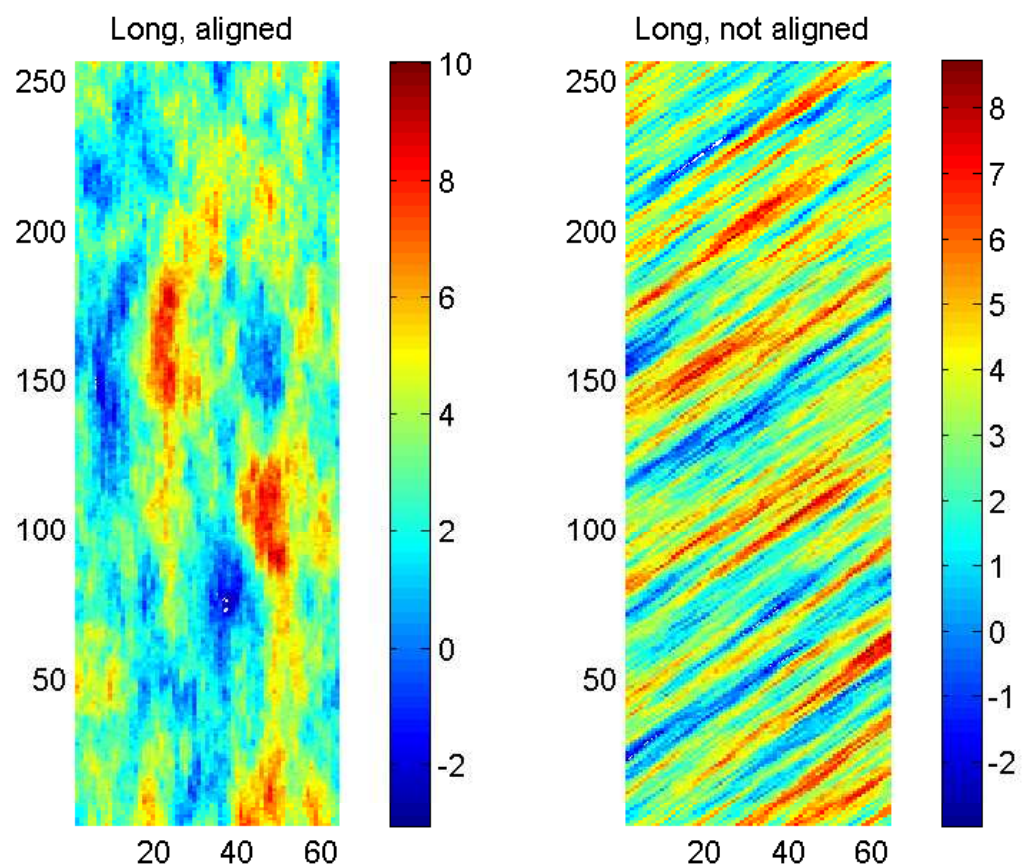

FIG. 4.1. Fine-scale permeability fields used as test cases in Section 4.1. Left: long correlation length, aligned to grid. Right: long correlation length, not aligned to grid. The grids contain $256 \times 64$ cells, with $\Delta x=\Delta y=1$.
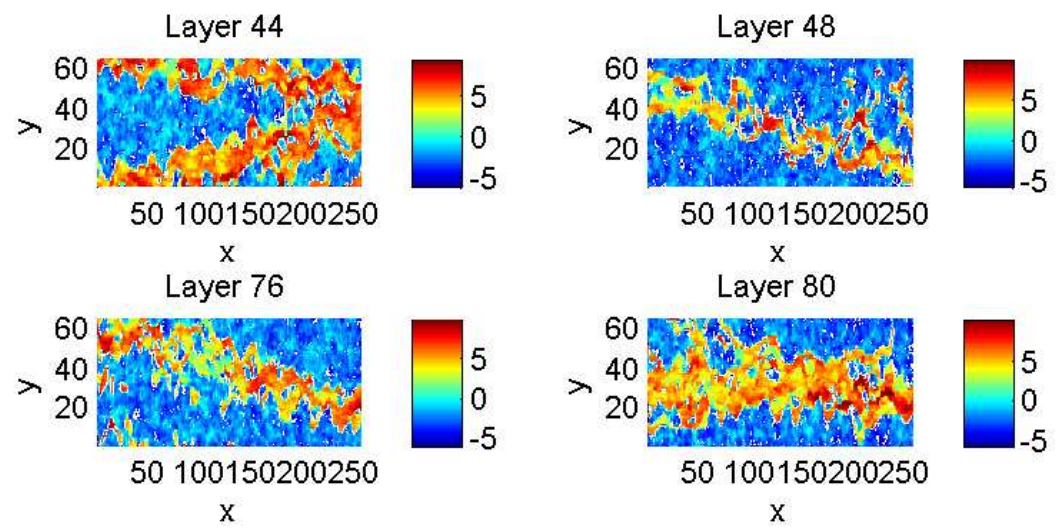

FIG. 4.2. Fine-scale permeability fields for modified version of SPE10 layers 44, 49, 76 and 80. The grids contain $256 \times 64$ cells, with $\Delta x=\Delta y=1$.

$p_{y}(x, 0)=p_{y}\left(x, L_{y}\right)=0$. For the second set, we prescribe $p(x, 0)=1$, $p\left(x, L_{y}\right)=0$, and $p_{x}(0, y)=p_{x}\left(L_{x}, y\right)=0$. These are the generic boundary conditions used for the global coarse solves in local-global upscaling, and also for the local fine solves in local or extended-local upscaling.

- Flow from each of the four corners of the domain. For example, for flow 
from the lower left corner, we prescribe $p(0, y)=\exp \left(-\left(2 y / L_{y}\right)^{4}\right)$ (shown in Figure 4.3), $p(x, 0)=\exp \left(-\left(2\left(L_{x}-x\right) / L_{x}\right)^{4}\right)$, and $p\left(L_{x}, y\right)=p\left(x, L_{y}\right)=0$. The boundary conditions for the other three corners are defined similarly.

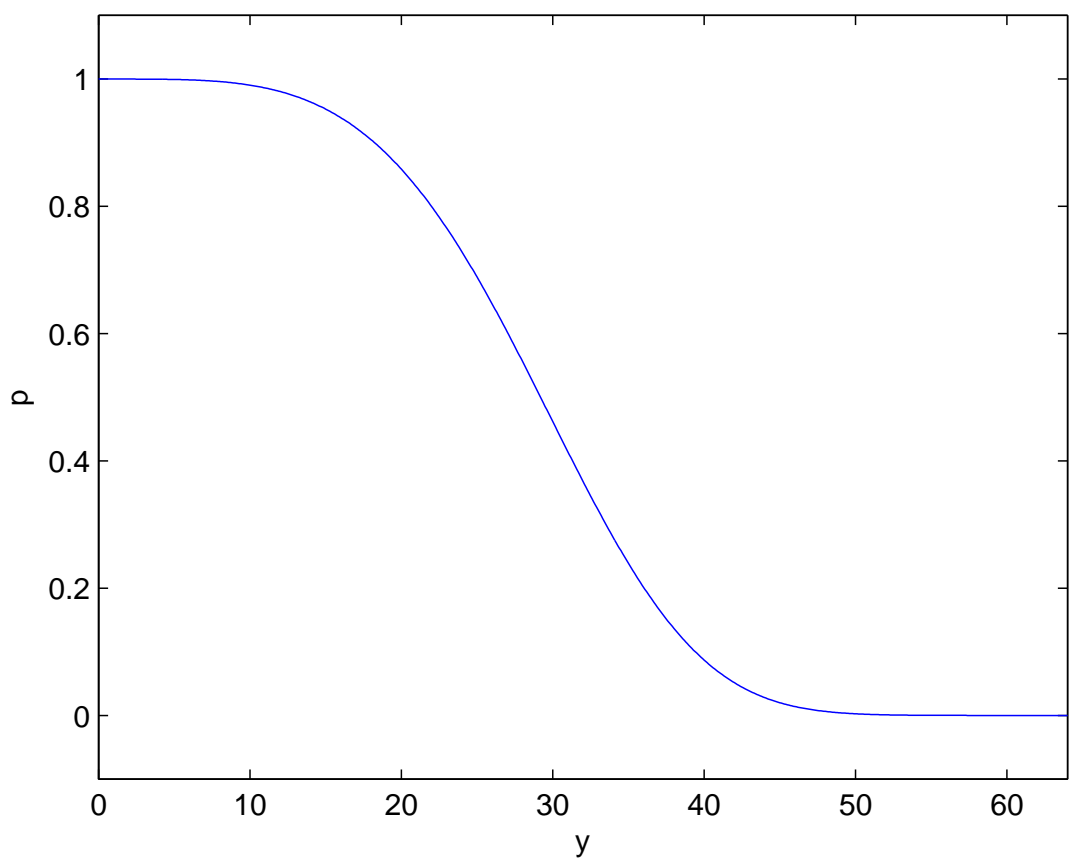

FIG. 4.3. The pressure $p(0, y)=\exp \left(-\left(2 y / L_{y}\right)^{4}\right)$ prescribed on the left boundary, for the set of boundary conditions in which flow emanates from the lower left corner of the domain.

We will compare the total flow to that obtained from a fine-scale solution, for each set of boundary conditions, and for each upscaling method. For cases generated from 2-point statistics, we present the mean and standard deviation of relative errors in total flow across all realizations. In selected cases, we will also examine the velocity fields, compared to the fine-scale velocity field averaged over each coarse grid cell.

4.2. Non-channelized cases. We first report results for the test cases involving permeability fields with long correlation lengths.

4.2.1. Long Correlation Length, Aligned with Grid. The relative errors in total flow are given in Table 4.2 for the 10 realizations. Clearly, both methods outperform EL. This is expected, and in agreement with what is reported in [7], as EL cannot accurately represent permeability contrasts at a scale larger than the coarse grid size. We see that LG and LG-W are quite comparable, due to the fact that the gradient-based weighting suggested in Section 3.2.4 is designed to behave like LG in regions where the flow is oriented with the grid, which is the case with these realizations.

4.2.2. Long Correlation Length, Not Aligned with Grid. Table 4.3 lists the relative errors in the total flow for the 10 realizations with long correlation lengths $\ell_{x}=75$ and $\ell_{y}=5$, not aligned to the grid. This time, both EL and LG perform 


\begin{tabular}{|l|r|r|r|r|r|r|r|r|}
\hline & \multicolumn{2}{|c|}{ Fine } & \multicolumn{2}{|c|}{ EL } & \multicolumn{3}{|c|}{ LG } & \multicolumn{3}{c|}{ LG-W } \\
\hline Flow & Mean & Std Dev & Mean & Std Dev & Mean & Std Dev & Mean & Std Dev \\
\hline In x-direction & 53.97 & 6.11 & 1.36 & 0.31 & 0.45 & 0.36 & 0.44 & 0.39 \\
\hline In y-direction & 53.97 & 6.11 & 0.50 & 0.19 & 0.58 & 0.22 & 0.63 & 0.19 \\
\hline From lower left & 18.83 & 6.00 & 1.00 & 0.56 & 1.17 & 0.71 & 1.16 & 0.78 \\
\hline From upper left & -19.62 & 7.67 & 1.58 & 0.72 & 1.55 & 0.84 & 1.50 & 0.73 \\
\hline From lower right & 16.97 & 5.69 & 1.39 & 0.69 & 1.09 & 0.66 & 1.16 & 0.62 \\
\hline From upper right & -17.63 & 6.36 & 0.80 & 0.42 & 0.80 & 0.46 & 0.85 & 0.40 \\
\hline
\end{tabular}

Mean percentage errors in total flow, and corresponding standard deviations, after applying various upscaling methods to ten realizations with long correlation length, aligned with the grid, using a $32 \times 16$ coarse grid.

poorly. LG-W has the best overall performance, and is quite accurate for all boundary conditions, except for the low-flow case in which flow is driven in the $x$-direction. Such sensitivity of the upscaling accuracy in weaker flow cases is fairly typical for TPFA methods on permeability fields that are not grid-aligned. In such cases, it is indeed preferable to go to MPFA methods, see, for example [23].

\begin{tabular}{|l|r|r|r|r|r|r|}
\hline & \multicolumn{2}{|c|}{ EL } & \multicolumn{2}{|c|}{ LG } & \multicolumn{2}{|c|}{ LG-W } \\
\hline Flow & Mean & Std Dev & Mean & Std Dev & Mean & Std Dev \\
\hline In x-direction & 6.38 & 4.58 & 22.10 & 5.26 & 29.82 & 5.91 \\
\hline In y-direction & 14.94 & 5.95 & 6.35 & 3.72 & 3.15 & 1.74 \\
\hline From lower left & 26.84 & 7.13 & 16.07 & 4.52 & 4.53 & 2.94 \\
\hline From upper left & 18.94 & 6.73 & 9.59 & 3.88 & 3.63 & 2.77 \\
\hline From lower right & 12.99 & 4.76 & 6.03 & 3.38 & 3.27 & 4.02 \\
\hline From upper right & 26.58 & 5.95 & 15.52 & 4.84 & 4.69 & 2.21 \\
\hline
\end{tabular}

Mean percentage errors in total flow, and corresponding standard deviations, after applying various upscaling methods to ten realizations with long correlation length, not aligned with the grid, using a $32 \times 16$ coarse grid.

Figure 4.4 shows portions of the velocity field computed by EL and LG-W. The portions chosen are those with the strongest flow. It can be seen from these figures that LG-W captures both the direction and magnitude of the fine-scale velocity vectors more accurately than EL, which yields velocity vectors that, locally tend to have a fixed orientation and not change direction as rapidly as the field generated by LG-W.

4.3. Channelized cases. Table 4.4 summarizes the results for layer 44 (see Figure 4.2 ) for the global flow, comparing multi-level local-global upscaling with the new transmissibility weighting (MLLG-W) to extended local upscaling on a Cartesian grid (EL), local-global upscaling on a Cartesian grid (LG), local-global upscaling with the new weighting on a Cartesian grid, (LG-W), and multi-level local-global upscaling without the new weighting (MLLG). We see that in all cases, the new transmissibility weighting improves the results significantly. Not only are the errors in total flow reduced strongly, process dependency is also greatly reduced. MLLG yields comparable accuracy to the Cartesian methods, while using a CCAR grid that contains significantly fewer cells due to coarsening in low-flow regions.

To demonstrate that both local-global upscaling and adaptivity contribute to accurate resolution of flow, we compare our algorithm to a modification of the algorithm 

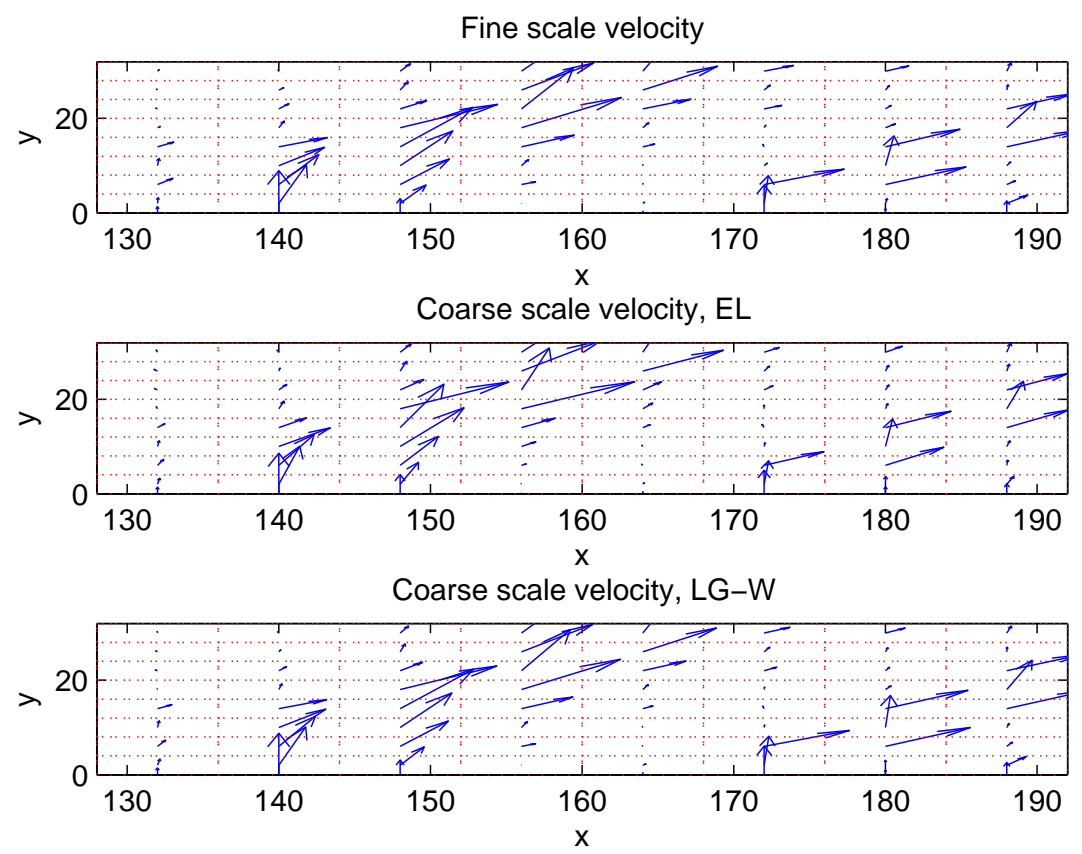

FIG. 4.4. Velocity fields for permeability field with long correlation length, not aligned with the grid, with flow in the $y$-direction. Top plot: fine-scale velocity, averaged over each cell of a $32 \times 16$ coarse grid. Bottom plot: coarse-scale velocity, obtained using method LG.

\begin{tabular}{|c|r|r|r|r|r|r|}
\hline Flow & $\begin{array}{r}\text { Fine-scale } \\
\text { total flow }\end{array}$ & \multicolumn{1}{c|}{ EL } & LG & LG-W & MLLG & MLLG-W \\
\hline In $x$-direction & 7.8544 & 13.68 & 3.55 & 3.61 & 0.09 & 3.59 \\
\hline In $y$-direction & 35.7709 & 7.20 & 2.68 & 3.57 & 2.31 & 3.51 \\
\hline From lower left & 7.4063 & 8.51 & 1.78 & 0.44 & 1.57 & 1.46 \\
\hline From upper left & -1.346 & 0.96 & 12.53 & 0.62 & 20.31 & 1.73 \\
\hline From lower right & 21.0316 & 3.95 & 3.97 & 6.54 & 3.40 & 5.93 \\
\hline From upper right & -28.0695 & 4.86 & 3.27 & 4.64 & 2.60 & 4.26 \\
\hline
\end{tabular}

Fine-scale total flow (first column) and percentage errors in total flow for modified SPE10 Layer 44 for various upscaling methods and boundary conditions. The EL, $L G$ and $L G-W$ methods use a $32 \times 16$ Cartesian grid, MLLG uses a 365-cell CCAR grid, and MLLG-W a 371-cell CCAR grid. The difference in these CCAR grids is due to the differences in transmissibility calculations, thus differences in local flow calculations, which impact the refinement criteria.

in which extended local upscaling is used for each CCAR grid, as opposed to using extended local only for the first inner iteration with the initial grid. Table 4.5 lists global flow errors for both algorithms. In all but two cases, local-global upscaling yields significantly more accuracy, even with fewer cells.

In Figure 4.5, we demonstrate the convergence of the global flow as the grid is refined, using the criteria defined in Section 3.2.3. We can see that for both generic global flow problems, with flow driven by pressure differences in the $x$-direction and 


\begin{tabular}{|l|r|r|}
\hline Flow direction & $\begin{array}{r}\text { \% error in global flow, } \\
\text { extended local, } \\
\text { 353-cell CCAR grid }\end{array}$ & $\begin{array}{r}\text { \% error in global flow, } \\
\text { local-global, } \\
\text { 371-cell CCAR grid }\end{array}$ \\
\hline In $x$-direction & 17.39 & 3.59 \\
\hline In $y$-direction & 7.18 & 3.51 \\
\hline From lower left & 8.25 & 1.46 \\
\hline From upper left & 1.22 & 1.73 \\
\hline From lower right & 5.31 & 5.93 \\
\hline From upper right & 6.05 & 4.26 \\
\hline
\end{tabular}

Total flow error from applying extended local upscaling and MLLG on CCAR grids, with various boundary conditions, to the modified SPE10 layer 44

$y$-direction respectively, convergence to the fine-scale global flow is obtained after just two passes of refinement. Furthermore, a subsequent local-global iteration on the final base grid converges immediately to the desired accuracy. The grids from the iterative refinement process are shown in Figure 4.6. Similar results are obtained for the other layers.
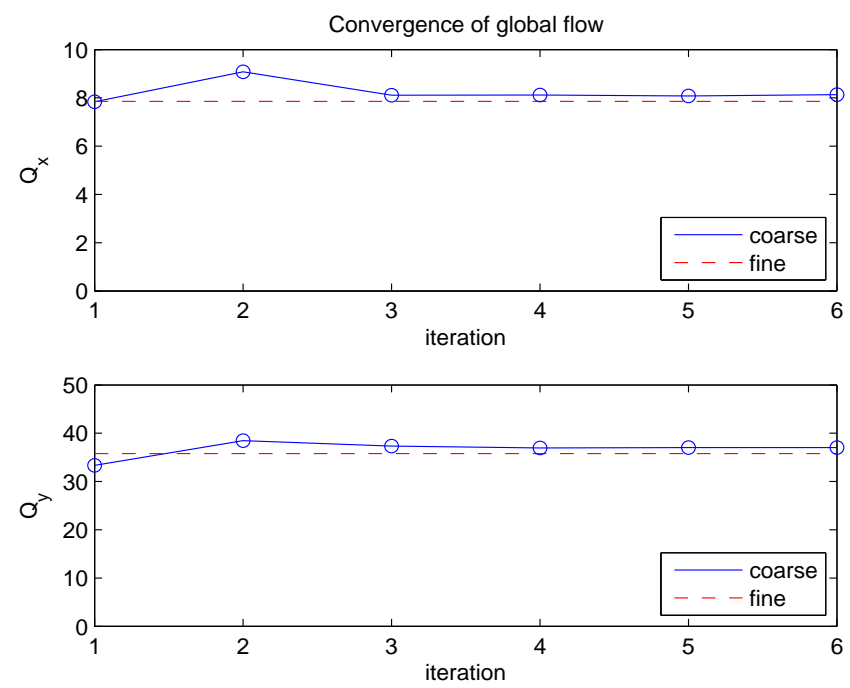

FIG. 4.5. Convergence of the global flow from the coarse-scale solutions to the pressure equation with permeability defined by a modified SPE layer 44, and generic boundary conditions. In both cases, the five outer iterations correspond to an initial iteration using extended local upscaling on a 32-cell Cartesian grid, three iterations of local-global upscaling on CCAR grids containing 32, 116, and 389 cells, respectively, followed by a final iteration of local-global upscaling on the 389-cell grid. Top plot: global flow in the $x$-direction. Bottom plot: global flow in the y-direction.

We repeat the comparisons of the upscaling methods with layer 48. Table 4.6 lists the errors in total flow for the same upscaling methods and boundary conditions. MLLG-W yields comparable or greater accuracy for all six boundary conditions. It is again interesting to note that for both Cartesian and CCAR grids, the new transmissibility weighting significantly improves accuracy for the highest-flow case (flow in the $x$-direction), without much loss of accuracy for the other boundary conditions, due 


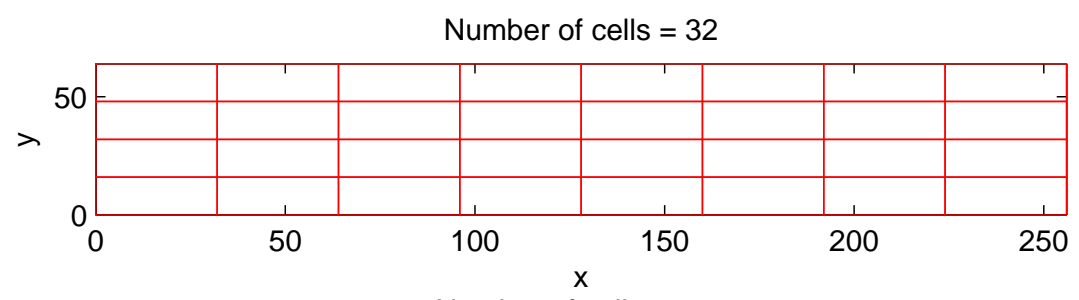

Number of cells $=104$

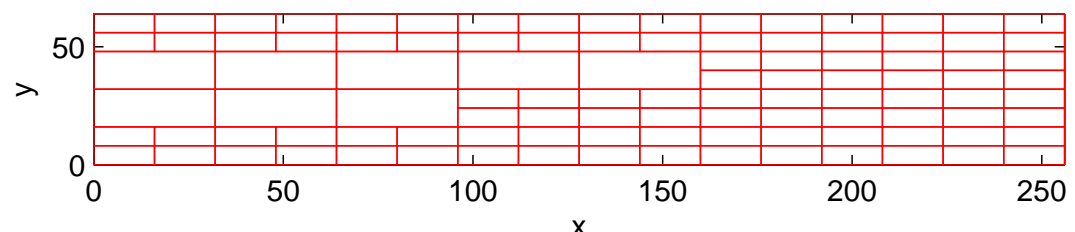

Number of cells $=371$

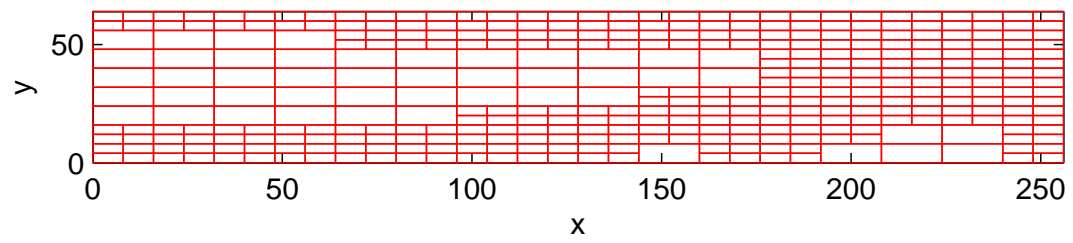

FIG. 4.6. Grids obtained by successive refinement, based on the distribution of the flux obtained from solutions of the pressure equation, with permeability defined by a modified SPE layer 44 and generic boundary conditions.

to its improved reduction of process dependency. Furthermore, applying the same upscaling method to a CCAR grid instead of a Cartesian grid results in improved accuracy in most cases for fewer grid cells. Finally, note the particularly dismal performance of extended local upscaling, compared to layer 44 above. It is important to remember that these results were obtained with a TPFA method.

\begin{tabular}{|c|r|c|r|r|r|r|}
\hline Flow & Fine scale & EL & \multicolumn{1}{c|}{ LG } & LG-W & MLLG & MLLG-W \\
\hline In $x$-direction & 3.1182 & 65.11 & 6.72 & 4.60 & 5.50 & 3.37 \\
\hline In $y$-direction & 1.6742 & 19.13 & 6.46 & 7.12 & 6.29 & 6.92 \\
\hline From lower left & 0.44651 & 36.38 & 9.34 & 7.80 & 7.14 & 5.13 \\
\hline From upper left & 1.2255 & 89.84 & 11.31 & 13.63 & 11.71 & 14.03 \\
\hline From lower right & -1.8434 & 87.43 & 13.31 & 16.17 & 14.76 & 17.03 \\
\hline From upper right & -0.27058 & 26.11 & 34.71 & 36.91 & 34.36 & 34.04 \\
\hline
\end{tabular}

Errors in total flow for modified SPE10 Layer 48, for various upscaling methods and boundary conditions. The EL, $L G$ and $L G$-W methods use a $32 \times 16$ Cartesian grid, MLLG uses a 383-cell $C C A R$ grid, and MLLG-W a 386-cell CCAR grid. The difference in these CCAR grids is due to the differences in transmissibility calculations, thus differences in local flow calculations, which impact the refinement criteria.

Figures 4.7 and 4.8 show a portion of the velocity field computed by LG-W and MLLG-W, respectively, for the case in which flow is driven in the $x$-direction. Both 
methods accurately resolve the fine-scale velocity, but MLLG-W does so with fewer cells.
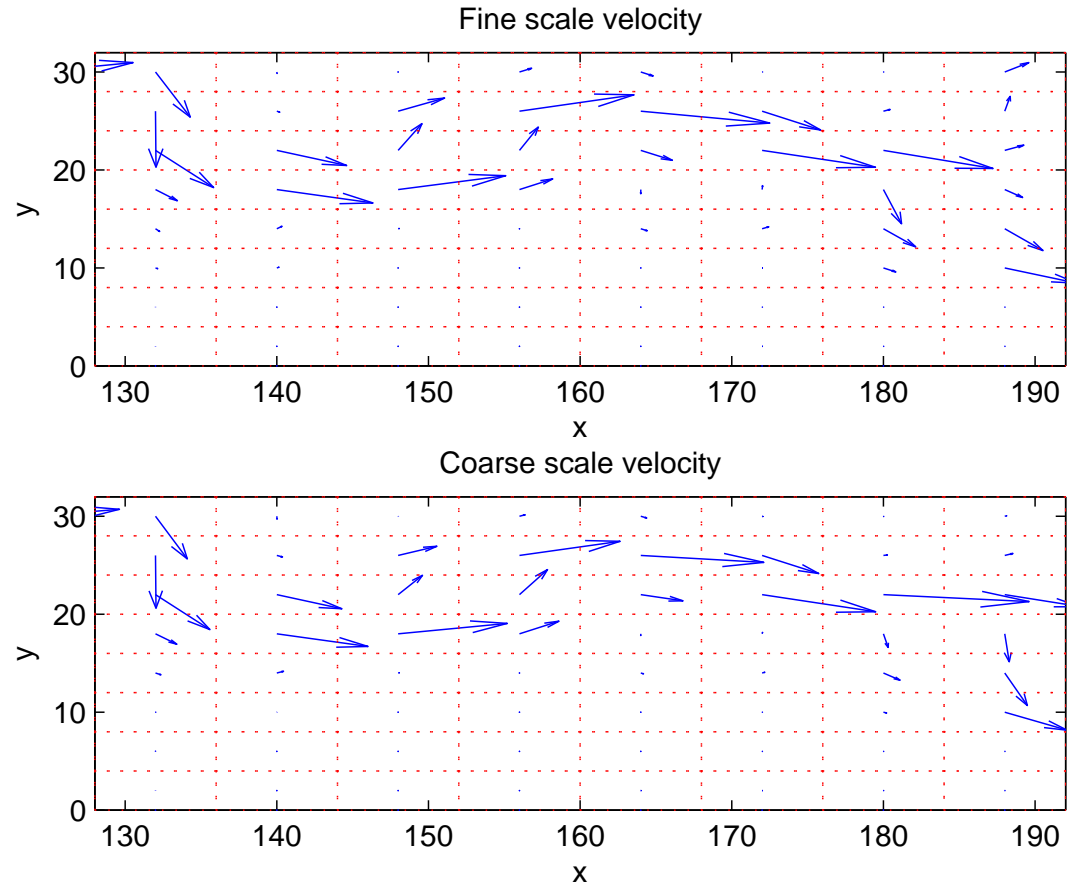

FIG. 4.7. Velocity fields for channelized domain (layer 48), with flow driven in the $x$-direction. Top plot: fine-scale velocity, averaged over each cell of a $32 \times 16$ Cartesian grid. Bottom plot: coarse-scale velocity, obtained using method $L G-W$.

Table 4.7 shows the percentage errors in total flow for layer 76 . This layer poses severe challenges to TPFA methods. Indeed, none of the methods are able to match the fine-scale flow with reasonable accuracy in the cases of flow from the upper left or lower right corner, so they are omitted here. For the remaining boundary conditions, we observe that (1) using a CCAR grid preserves the accuracy obtained using a Cartesian grid with more cells, and (2) gradient-based weighting reduces the process dependency for both Cartesian and CCAR grids, making MLLG-W the best allaround choice.

\begin{tabular}{|c|r|r|r|r|r|r|}
\hline Flow & Fine scale & \multicolumn{1}{|c|}{ EL } & \multicolumn{1}{c|}{ LG } & LG-W & MLLG & MLLG-W \\
\hline In $x$-direction & 4.2935 & 32.91 & 5.69 & 3.39 & 5.93 & 3.99 \\
\hline In $y$-direction & 1.5661 & 2.46 & 4.20 & 4.63 & 3.83 & 4.52 \\
\hline From lower left & 0.42436 & 38.23 & 36.81 & 26.79 & 33.69 & 26.25 \\
\hline From upper right & -0.099821 & 9.30 & 16.96 & 19.46 & 17.26 & 20.50 \\
\hline
\end{tabular}

Errors in total flow for modified SPE10 Layer 76, for various upscaling methods and boundary conditions. The EL, $L G$ and $L G$-W methods use a $32 \times 16$ Cartesian grid, $L G$-CCAR uses a 425-cell CCAR grid, and MLLG uses a 422-cell CCAR grid.

Table 4.8 shows the percentage errors in total flow for the same upscaling methods 

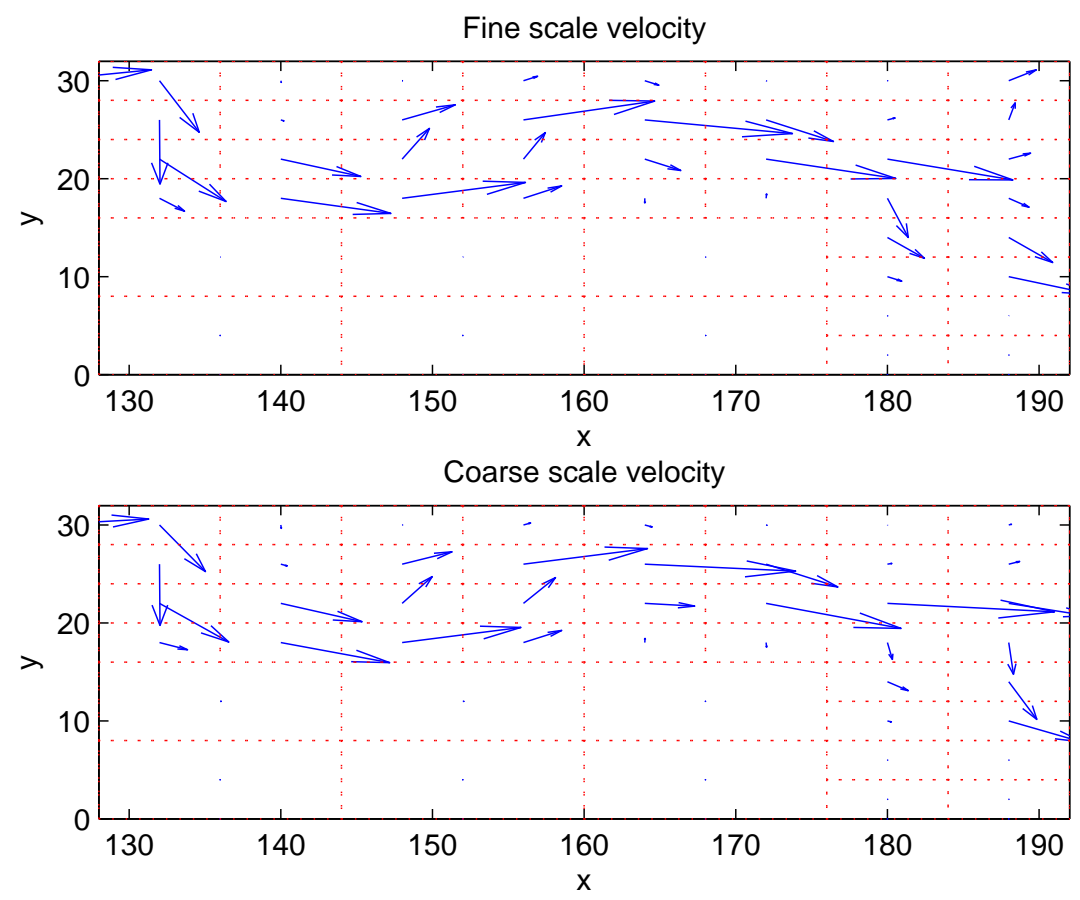

FIG. 4.8. Velocity fields for channelized domain (layer 48), with flow driven in the $x$-direction. Left plot: fine-scale velocity, averaged over each cell of a 371-cell CCAR grid. Bottom plot: coarsescale velocity, obtained using $M L L G-W$.

for layer 80. In this layer, the main channel is oriented along the grid. Because of this orientation, the upscaling methods that employ gradient-based weighting do not provide as much benefit, but they still exhibit reduction in process dependency. In particular, note the smaller errors for the low-flow cases.

\begin{tabular}{|c|r|r|r|r|r|r|}
\hline Flow & Fine scale & \multicolumn{1}{|c|}{ EL } & \multicolumn{1}{c|}{ LG } & LG-W & MLLG & MLLG-W \\
\hline In $x$-direction & 14.6725 & 12.23 & 6.22 & 4.87 & 5.88 & 6.10 \\
\hline In $y$-direction & 3.0696 & 4.07 & 0.11 & 1.24 & 0.02 & 1.29 \\
\hline From lower left & 13.2104 & 6.81 & 6.69 & 6.77 & 4.28 & 6.21 \\
\hline From upper left & -8.9824 & 6.53 & 5.02 & 7.08 & 0.37 & 4.60 \\
\hline From lower right & -7.8925 & 5.31 & 9.66 & 6.83 & 10.05 & 8.28 \\
\hline From upper right & -0.60702 & 0.30 & 17.33 & 12.39 & 15.27 & 14.24 \\
\hline
\end{tabular}

Errors in total flow for modified SPE10 Layer 80, for various upscaling methods and bounday conditions. The EL, LG and $L G$-W methods use a $32 \times 16$ Cartesian grid, MLLG uses a 431-cell $C C A R$ grid, and $M L L G-W$ uses a 419-cell CCAR grid.

5. Discussion. This work shows that it is possible to obtain good upscaling accuracy on challenging permeability fields, such as the channelized cases used in this paper, on Cartesian adapted grids (CCAR grids) and two-point flux approximations (TPFA) using the proposed multi-level local-global method (MLLG). We compared 
global flow errors for channelized systems, which are generally challenging for upscaling methods, produced by upscaled CCAR grids to errors produced by local-global upscaling on Cartesian grids of similar density. In [7] it was shown that the uniform local-global method on these channelized systems strongly outperformed local or extended local upscaling methods. For the generic boundary conditions used in the upscaling process the upscaled CCAR grids cut these global flow errors again, often as much as $50 \%$ or more. Process dependency is also reduced significantly. These results suggest that refinement is an effective means to control upscaling errors and reduce process dependency. A large contributor to the improved accuracy presented in this paper is the new gradient-based weighting of transmissibilities, which we strongly recommend for local-global upscaling strategies.

We are especially encouraged by the accuracy we obtain with our method in the local flow. The combination of adaptivity and local-global upscaling retains important connected flow paths and accurately compute local flow directions and speeds. This is promising for displacements with high mobility contrasts, such as gas injection processes, where the mobile fluid will seek these high permeability paths. We are implementing the CCAR methods in a compositional solver to further investigate this potential benefit.

The computational overhead of MLLG is relatively low, because global solves are performed on coarse grids, the sizes of extended local regions are limited, and generally very few steps are required for the local-global iteration to converge.

The effectiveness of MLLG is good news, as it means that it is perhaps not as essential to align computational grids, thus simplifying gridding strategies, or to always use more expensive multi-point flux approximations. Multi-point flux approximations are necessary in cases with very strong permeability tensor effects and/or weak flows. If such areas are local and can be identified, this opens the possibility of using adaptive stencils that employ TPFA where possible and revert to MPFA in the critical areas. One such method is introduced in [23], and other related work can be found in $[2]$.

Future work will include the investigation of other possible adaptivity criteria, including criteria for coarsening, and alternative methods of handling occurrences of negative transmissibilities. The upscaling approach presented here, while suitable for CCAR grids, can just as easily be applied to other types of grids. Future work will include generalization of our algorithm to other gridding strategies such as patched refinements, unstructured grids, or curvilinear grids.

6. Conclusions. The following main conclusions can be drawn from this study:

- For highly heterogeneous (e.g., channelized) systems, the proposed integration of grid adaptivity and upscaling is shown to consistently provide more accurate coarse-scale models for global flow, relative to reference fine-scale results, than do existing upscaling techniques using TPFA discretizations, including local-global upscaling, applied to uniform grids of similar densities.

- Adaptation allows the use of a coarse-scale model that contains fewer grid points than would be required when using a Cartesian grid to achieve the same accuracy during simulation.

- Apart from more accurate global flow results, the proposed method also computes improved local flow results. Connected high permeability flow paths are preserved, and local velocity directions and magnitude in these important paths are computed with good accuracy.

- We developed a refinement indicator based on local fluxes that guides the grid 
adaptation process during upscaling and further reduces solver and upscaling errors.

- Process dependency is strongly reduced, that is, the approach computes accurate global flow results also for flows driven by boundary conditions different from the generic boundary conditions used to compute the upscaled parameters.

- Upscaling accuracy can be significantly improved and process dependency reduced with the use of a gradient-based weighting of transmissibilities computed from multiple local flow problems in the local-global method.

Acknowledgments. This work was supported by DOE Grant DE-FC26-04NT15530M001, Computer Modeling Group, and our SUPRI-C affiliates. The authors would also like to thank Jonas Nilsson and Rami Younis for their contributions of crucial code and helpful discussion.

\section{REFERENCES}

[1] Aavatsmark I, Barkve T, Mannseth T. Discretization on Non-Orthogonal, Quadrilateral Grids for Inhomogeneous, Anisotropic Media. Journal of Computational Physics, 127:2-14, 1996.

[2] Aavatsmark I, Eigestad GT and Nordbotten JM. A compact MPFA method with improved robustness. Proceedings of the 10th European Conference on the Mathematics of Oil Recovery, 2006.

[3] Berger MJ, Colella P. Local adaptive mesh refinement for shock hydrodynamics. Journal of Computational Physics, 82:64-84, 1989.

[4] Berger MJ, Oliger JE. Adaptive Mesh Refinement for Hyperbolic Partial Differential Equations. Journal of Computational Physics, 53:484-512, 1984.

[5] Bourgeat A. Homogenized Behavior of Two-Phase Flows in Naturally Fractured Reservoirs with Uniform Fractures Distribution. Computational Methods in Applied Mechanics and Engineering, 47:205-16, 1984.

[6] Chen Y, Durlofsky LJ. Adaptive Local-Global Upscaling for General Flow Scenarios in Heterogeneous Formations. Transport in Porous Media 62:157-185, 2006.

[7] Chen Y, Durlofsky LJ, Gerritsen MG, Wen XH. A coupled local-global upscaling approach for simulating flow in highly heterogeneous formations. Advances in Water Resources, 26:10411060, 2003

[8] Christie MA, Blunt MJ. Tenth SPE comparative solution project: a comparison of upscaling techniques. SPE Reservoir Evaluat Eng 2001;4:308-17.

[9] Deutsch C, Journel AG. GSLIB: Geostatistical Software Library and User's Guide, 2nd. Edition, Oxford Press, 1998.

[10] Durlofsky LJ. Numerical Calculation of Equivalent Grid Block Permeability Tensors for Heterogeneous Porous Media. Water Resources Research, 27:699-708, 1991.

[11] Edwards MG. Elimination of Adaptive Grid Interface Errors in the Discrete Cell Centered Pressure Equation. Journal of Computational Physics, 126:356-372, 1996.

[12] Edwards MG, Rogers CF. Finite Volume Discretization with Imposed Flux Continuity for the General Tensor Pressure Equation. Computational Geosciences, 2:259-290, 1998.

[13] Gautier Y, Blunt MJ, Christie MA. Nested Gridding and Streamline-Based Simulation for Fast Reservoir Performance Prediction. SPE Reservoir Simulation Symposium, 1999, SPE 51931.

[14] Gerritsen MG, Jessen K, Mallison BT, Lambers JV. A Fully Adaptive Streamline Framework for the Challenging Simulation of Gas-Injection Processes. SPE ATC, 2005, SPE 97270.

[15] Gerritsen MG, Lambers JV. Solving Elliptic Equations in Heterogeneous Media using Cartesian Grid Methods with Anisotropic Adaptation. To be submitted to Journal of Computational Physics, 2007.

[16] Ham FE, Lien FS, Strong, AB. A Cartesian Grid Method with Transient Anisotropic Adaptation. Journal of Computational Physics, 179:469-494, 2002.

[17] He C. Structured Flow-Based Gridding and Upscaling for Reservoir Simulation. PhD Thesis, Department of Petroleum Engineering, Stanford University.

[18] Henson VE, Yang UM. BoomerAMG: a Parallel Algebraic Multigrid Solver and Preconditioner. Applied Numerical Mathematics, 41:155-77, 2002. 
[19] Holden L, Nielsen BF. Global Upscaling of Permeability in Heterogeneous Reservoirs: the Output Least Squares (OTL) Method. Transport in Porous Media, 40:115-43, 2000.

[20] Hornung R, Trangenstein J. Adaptive Mesh Refinement and Multilevel Iteration for Flow in Porous Media. Journal of Computational Physics, 136:522-545, 1997.

[21] Kippe V, Aarnes JE, Lie KA. A Comparison of Multiscale Methods for Elliptic Problems in Porous Media Flow. To be published in Computational Geosciences, Special issue on Multiscale Methods, 2007.

[22] Lee SH, Tchelepi HA, Jenny P, DeChant LJ. Implementation of a Flux-Continuous FiniteDifference Method for Stratigraphic, Hexahedron Grids. SPE Journal, 7:267:277, 2002.

[23] Lambers JV, Gerritsen MG, Mallison BT. Accurate Local Upscaling with Variable Compact Multi-point Transmissibility Calculations. To be published in Computational Geosciences, Special issue on Multiscale Methods, 2007.

[24] Mittal R, Iaccarino, G. Immersed Boundary Methods. Annual Review of Fluid Mechanics, 37:239-61, 2005.

[25] Nilsson J, Gerritsen MG, Younis RM. A Novel Adaptive Anisotropic Grid Framework for Efficient Reservoir Simulation. Proc. of the SPE Reservoir Simulation Symposium, 2005, SPE 93243.

[26] Nordbotten JM, Aavatsmark I, Eigestad GT. Monotonicity of Control Volume Methods. Submitted to Numerische Mathematik, 2006.

[27] Pickup GE, Ringrose PS, Jensen JL, Sorbie KS. Permeability Tensors for Sedimentary Structures. Mathematical Geology, 26:227-50, 1994.

[28] Sammon, P H, "Dynamic Grid Refinement and Amalgamation for Compositional Simulation", SPE RSS, 2003, SPE 79683

[29] Trangenstein J, Bi Z. Large Multi-Scale Iterative Techniques and Adaptive Mesh Refinement for Miscible Displacement Simulation. SPE/DOE Improved Oil Recovery Symposium, 2002, SPE 75232 .

[30] Watson DF. Contouring: A guide to the analysis and display of spacial data. Pergamon, 1994.

[31] Wen XH, Durlofsky LJ, Edwards MG. Use of Border Regions for Improved Permeability Upscaling. Mathematical Geology, 35:521-547, 2003.

[32] Younis RM, Caers J. A Method for Static-based Upgridding. Proc. of the 8th European Conference on the Mathematics of Oil Recovery, Sept. 2002 